\title{
DIC measurement of the full strain field in a tiled laminate to determine local and global stiffness properties
}

\author{
Jordi UYTTERSPROT'1,a, Wouter DE CORTEE ${ }^{1, b}$, Wim VAN PAEPEGEM ${ }^{2, c}$ \\ ${ }^{1}$ Department of Structural Engineering, Faculty of Engineering and Architecture, Ghent University, \\ Valentin Vaerwyckweg 1, 9000 Ghent, Belgium \\ ${ }^{2}$ Department of Materials, Textiles and Chemical Engineering, Faculty of Engineering and \\ Architecture, Ghent University, Tech Lane Ghent Science Park 903, 9052 Zwijnaarde, Belgium \\ aJordi.Uyttersprot@UGent.be, bWouter.DeCorte@UGent.be, ‘Wim.VanPaepegem@UGent.be
}

Keywords: Tiled laminate, FRP composite, DIC

\begin{abstract}
Tiled laminates (TL) are bio-inspired oblique layered materials, composed of individual stacked plies in a tiled fashion, like the tiles on a slate-roof are positioned, and joined together with a polymer to form a rigid oblique layered structural material. This concept of an oblique layered buildup has great potential in different sectors (i.e. bridge building, offshore construction, ship building, lock gates, fire resistance panels, ...) as it allows manufacturing of composite plates from tiled strips of fabric which opens up the door for robotised production of panels of any shape and size. Because this is a relatively new concept, less is known about the mechanical properties of the material such as the strength, the local and global stiffness and the fracture mechanics properties. Further, the classic laminate theory does not fully describe a TL as opposed to a plane-parallel laminate. Although available, experimental data is needed to validate, calibrate and corroborate the numerical finite element (FE) models. In previous research, the longitudinal stiffness was described by strain gauges and extensometers, leading to scatter/variation in the results due to the specific location of the measurement equipment on the laminate, which cannot be justified by the FE models. This paper studies the full strain field in a TL under uniaxial tension by digital image correlation (DIC) and compares the results to the numerical FE models. As a guidance, ASTM D3039 was used to perform the tensile test even though the specimens are not symmetric or balanced as prescribed by the standard. A fine speckle pattern is applied to the specimen and a well-lit environment is created for the DIC measurement. The results show that the strains along a path in the FE models on the top and bottom of the tiled laminate show large variations and that the peak values at the free edges in the FE models need to be averaged out over a relatively large distance. Finally, the tests allow for good local and global stiffness characterisation of a TL.
\end{abstract}

\section{Introduction}

The concept of tiled layering to obtain special properties is new and can be applied on almost every scale. From molecular scale, e.g. oblique layered graphene, to macro scale, e.g. oblique layered modular ship sections, bridges/bridge decks, offshore and shipping applications, fire resistant panelling, etc. The concept of tiled composites (TC) can be implemented in a variety of different ways and with different types of materials, in fact any composite material in the broadest sense can be used [1]. From a pure technical perspective, the essential material properties of this type of laminate remain unknown. Plane-parallelism (PP), where the dry fabric layers are stacked parallel to each other, of the composite reinforcement is the basic hypothesis of the classical laminate theory. Since the classical laminate theory calculates the laminate stiffness matrix (ABD-matrix), it cannot accurately reflect the stiffness constants of a tiled laminate (TL). In practice, mechanical properties for design of plates fabricated in this way are currently based on tests [2]. Intuitively, the reader would also expect that tiled laminates, when loaded in tension, would fracture by shearing off in between the tiled plies, but preliminary tests show that this is not the case. The fracture is still more or less perpendicular to the loading direction, as schematically indicated in Figure 1, but at a reduced strength compared to the PP laminate. 
The TC differ greatly from the classical traditional PP way of constructing composites and the classical approaches to testing and modelling are insufficient or invalid. Therefore, the first step is to acquire a thorough understanding of the mechanics of this type of material through experimental

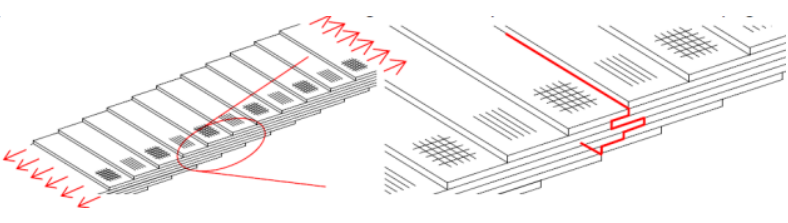

Figure 1: Typical failure of a laminate in tension testing. Numerical finite element (FE) models are available but these need to be validated, calibrated and corroborated by experimental data. In previous research, the longitudinal stiffness was described by strain gauges and extensometers, leading to scatter/variation in the results, due to the specific location of the measurement equipment on the laminate, which cannot be justified by the FE models. This paper studies the full strain field in a tiled laminate under uniaxial tension by digital image correlation (DIC) and compares the results to the numerical FE models. In the first part of this paper, the materials and methods are presented, and the experimental results are reported. In the second part of this paper, the test results are related to numerical results from a finite element calculation using Abaqus as numerical tool. Finally, conclusions are drawn.

\section{Materials and methods}

In this study, experimental input was gathered from uniaxial tensile testing and was suitably instrumented using digital image correlation (DIC). For this an Instron 5800R electro-mechanical tensile machine equipped with a $100 \mathrm{kN}$ load cell is used and the measurement of the displacement of the test specimen is achieved by a Point Grey DIC camera set.

Uniaxial tensile test - This test method determines the in-plane tensile properties of polymer matrix composite materials reinforced by high-modulus fibres. As a guidance, ASTM D3039 was used to perform the tensile test even though the TL specimen are not symmetric or balanced as prescribed by the standard. During this test, a thin flat strip of the laminate having a constant rectangular cross section is mounted in the grips of the mechanical testing machine and monotonically loaded in tension. The ultimate strength of the material can be determined from the maximum load carried at failure. Because the coupon strain is obtained from the displacement, monitored with DIC, the stress-strain response of the material can be determined, from which the ultimate tensile strain, tensile modulus of elasticity and Poisson's ratio can be derived. [3]

$D I C$ - Digital Image Correlation is an innovative non-contact optical technique for measuring strain and displacement [4]. The technique compares digital photographs of the surface of a test sample at different stages of deformation during the test. By tracking blocks of pixels, called subsets, the system can measure surface displacement and build up full field 2D and 3D deformation vector fields and derived from this strain maps. For DIC to work effectively, the subsets need to be random an unique with a range of contrast and intensity levels. For this reason a random speckle pattern is applied to the surface of the samples, using a low viscosity spray paint to not cover any newly created cracks in the specimen. To enlarge the contrast of the speckle pattern, first a thinly applied white background is used before the random black speckle pattern is applied. A high frame rate $(5 \mathrm{~Hz})$ is used for the cameras during the tests to obtain multiple photos

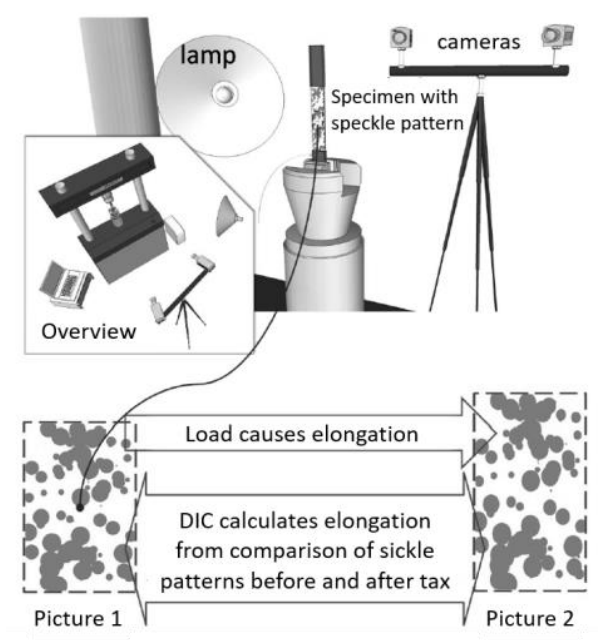

Figure 2: DIC setup each second which results in an underexposed image. Hence an extra light source is added to the DIC setup to allow more light to enter the cameras, bringing up the exposure of the image gaining well-lit and useable images for the software to process. Further the DIC setup (Figure 2) includes two cameras. One is placed perpendicular to the speckle pattern, creating a 2D displacement image of the 
specimen, while the other is placed at an angle to get the out of plane displacements. Both cameras are reciprocally synchronised to take pictures at the same time and to the test equipment to obtain a readout of the force at the exact moment a picture is taken.

Samples - The samples are composed of multiple E-glass fibre unidirectional (UD) plies and infused with polyester resin. This polyester resin is an unsaturated synthetic resins converted to a solid by cross-linking chains using a catalyst, methyl ethyl ketone peroxide (MEKP). During the production of the samples, $2 \%$ MEKP was used in combination with the polyester resin to infuse the GFRP TL plates. The specimen consists of repetitive $150 \mathrm{~mm}$ long fabric stacks, each consisting of four individual plies. In the direction of the specimen each stack contains two $0^{\circ}$-plies, one $45^{\circ}$-ply and one $-45^{\circ}$-ply ([0/45/-45/0]). These stacks are arranged in a tiled fashion with a predefined overlap length to produce a TL with a specific stacking angle. The plane-parallel (PP) reference sample consists of three stacks with the same build-up as described above, but who are stacked parallel to each other. The laminate plates, with dimensions of 300x300mm, are infused using a vacuum assisted resin transfer moulding (VARTM) technique. After curing they are cut by a water cooled diamond grinding wheel to the prescribed coupon size as stated in ASTM D3039, with a length of 250mm and a width of $25 \mathrm{~mm}$. The thickness of the test specimens varies depending on the build-up and the overlap length. At least five specimens per laminate were tested to acquire statistically significant data. Different specimen geometries and build-ups (Figure 3) were tested during this research.

After curing they are cut by a water cooled diamond grinding wheel to the prescribed coupon size as stated in ASTM D3039, with a length of $250 \mathrm{~mm}$ and a width of $25 \mathrm{~mm}$. The thickness of the test specimens varies depending on the build-up and the overlap length
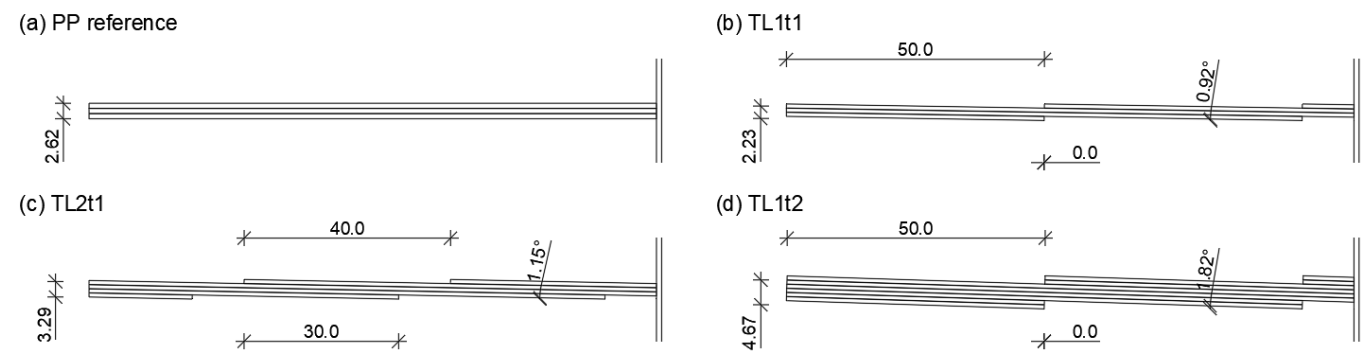

(d) TL1t2
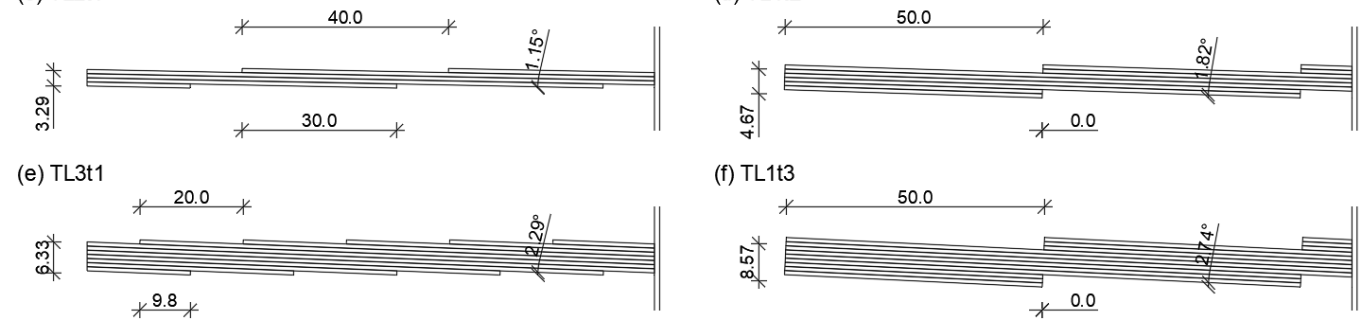

Figure 3: Geometry of the test specimen

Two techniques were used to produce different stacking angle in the tiled laminates. In the first technique the thickness of a single stack was altered by doubling or tripling the build-up (indicated with TL1), while in the second technique the overlap length of the stacks was changed (indicated with TL2 and TL3) resulting in a gradual transition between the different stacks. It should be noted that changing the overlap length of the stacks also enlarges the overall thickness of the specimen. The overall thickness indicated in Figure 3 is the average thickness of the five tested specimen out of a single manufactured plate, measured over three different places along the length of the specimen. The specimens listed as TL1 have a consistent overlap length of $50 \mathrm{~mm}$ for the stacks, but the thickness of a single stack is varied indicated by the suffix $\mathrm{t} 1, \mathrm{t} 2$ and $\mathrm{t} 3$ respectively one time the original thickness of the stack $([0 / 45 /-45 / 0])$, two times the thickness $\left([0 / 45 /-45 / 0]_{2}\right)$ and three times the thickness $\left([0 / 45 /-45 / 0]_{3}\right)$. Since the overlap length of $50 \mathrm{~mm}$ is a multiple of the total length of the stacks $(150 \mathrm{~mm})$, an abrupt transition is created between the different stacks as seen in Figure 3 (b), (d) and (f). For the specimens TL2t1 and TL3t1 the overlap length of the stacks is changed to 40mm and $20 \mathrm{~mm}$ respectively, resulting in a gradual transition. The PP reference sample, with a stacking angle of $0^{\circ}$, will be used to set a reference for the results of the global and local stiffness and the first and total failure strength of the TL's. 


\section{Results}

The DIC measurements indicate that the stiffness along a centreline in the length of the specimen changes in every position, which means that the strain value will be different on every point of the surface of the test specimen. This makes a point measurement, with a strain gauges or extensometer, on the surface of a TL very unreliable in order to determent the stiffness value of the laminate, something which could be concluded from previous research. In the present study, the full strain field of the specimen is captured, using a DIC measurement, resulting in a better representation of the global and local stiffness of the TL's. From that, the global stiffness and strength at first and total failure in function of the stacking angle for the PP reference sample (with a stacking angle of $0^{\circ}$ ) and the different TL's are derived.

Local stiffness - Figure 4 presents the local Young's modulus along the centreline of the different laminates for a set of average strains. The average strains are taken along the length of the centreline for a set of time periods. The time periods are set every 20s starting from the moment when at least $1000 \mu \varepsilon(0.1 \%)$ is achieved in order to have a good strain distribution along the length of the specimen. This interval is maintained until first failure of the specimen, because at the location of failure the speckle pattern will be destroyed in such a way that no correlation of the pattern is still possible. This results in a zero value for the strain at the location of failure, which does not correspond with the reality as, conversely there will be a large displacement at that location. Consequently the set of average strains for the different specimen can differ in the graphics of Figure 4.
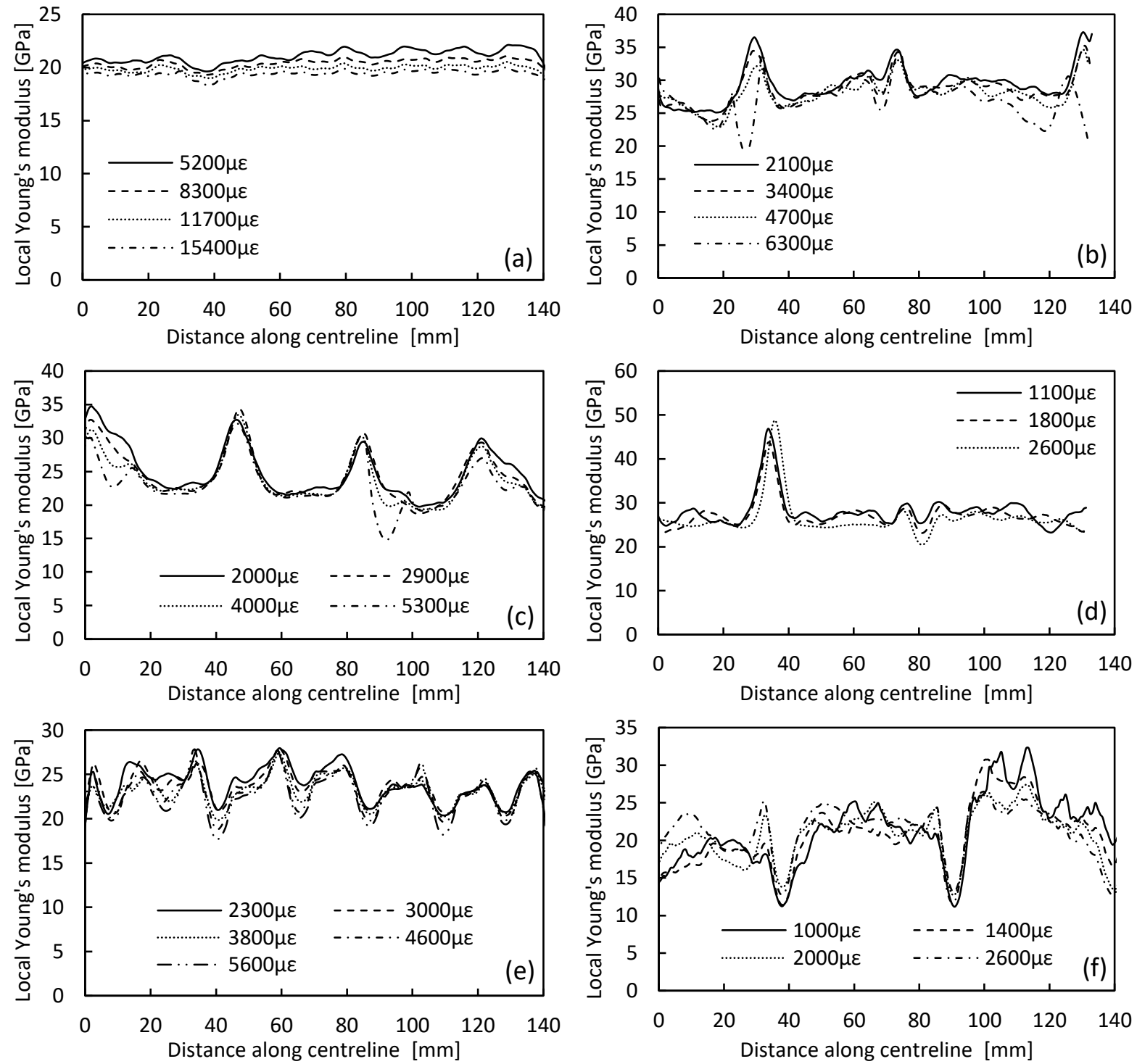

Figure 4: Young's modulus along centreline specimen PP (a), TL1t1 (b), TL2t1 (c), TL1t2 (d), TL3t1 (e) and TL1t3 (f) 
Figure 4 clearly shows the overlap locations in the TL's, where there is a peak or valley in the curves, and between two overlaps the stiffness value remains consistent such as in the PP reference sample. It can also be seen that to the end of the test (during the last time interval in the graphs), when failure is occurring, the stiffness at the location of failure will reduce. This reduction in stiffness is primarily seen at the overlap locations, since the onset of failure mainly starts at a free edge.

Global stiffness - Figure 5 presents the average stress-strain diagram of the PP reference sample and the different TL specimens. Here the stress is calculated by dividing the measured tensile force of the tensile machine by the averaged section along the length of the specimen, while the strain is directly derived from the DIC measurements averaged over the full length of the specimen. It should be noted that only the elastic section until the first failure is displayed, meaning that the maximal stress in this diagram does not correspond with the total tensile strength of the laminate specimen.

Using Hooke's law, the average Young's modulus of the laminates is calculated, after which the relative stiffness with respect to the stiffness of the PP reference sample is plotted as a function of the stacking angle in Figure 6. In this graph the relative strength until first $(\mathrm{FF})$ and total failure $(\mathrm{TF})$ with respect to the PP reference sample is also plotted. A negligible decline in stiffness can be detected between the PP reference sample and the different TL's on the one hand, while on the other hand a significant decrease in strength until FF and

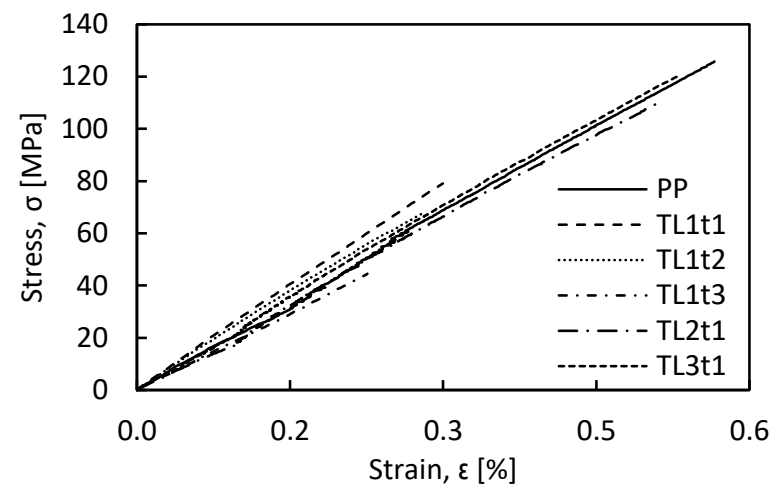

Figure 5: Stress-Strain diagram PP and TL's

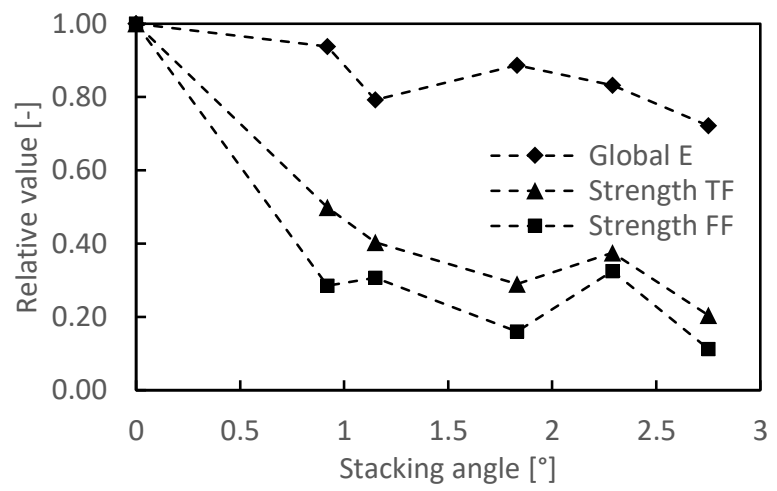

Figure 6: Relative stiffness in function of the stacking angle $\mathrm{TF}$ is observed when the stacking angle deviates from $0^{\circ}$ (PP reference sample). It is clear that the stacking angle has a small effect on the global stiffness of the specimen since a PP laminate is only between 10 to $20 \%$ stiffer then the TL's. A different conclusion can be made in relation with the strength of the specimen untill TF as a PP specimen is approximate 40 to $50 \%$ stronger than a TL.

Failure mechanism - Figure 7 illustrates the failure mechanisms of the PP reference sample (left) and two of the TL's (middle and right). As expected, fibre failure accompanied by an explosive failure event is the dominant failure mechanism for the PP reference sample. Comparing this to the TL's, failure occurred by shear between the different stacks and this for both the TL1t1 with a small thickness and an straight overlap as for TL3t 1 with a larger thickness and a more gradual overlap.

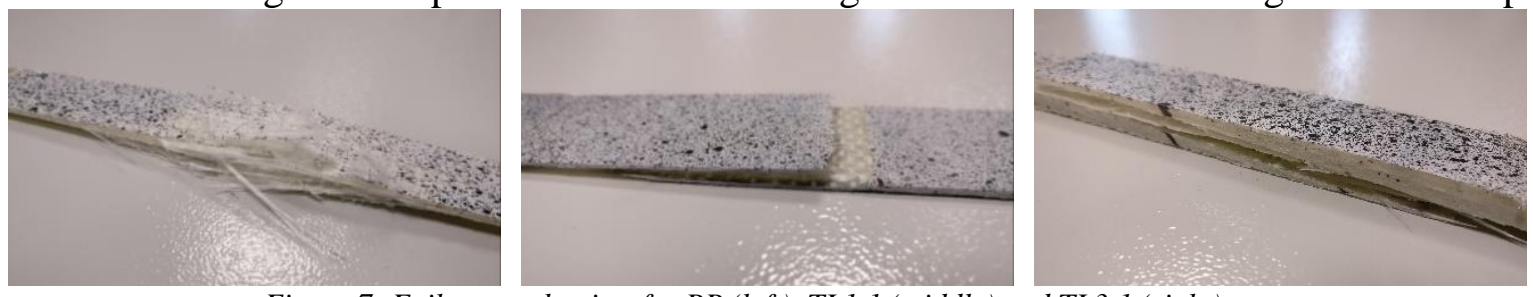

Figure 7: Failure mechanism for PP (left), TL1t1 (middle) and TL3t1 (right)

Besides, the failure event is slightly different for the two build-up techniques. In the first case (TL1) failure occurred more gently and could easily be foreseen while in the second case (TL2 and TL3) the failure occurred very suddenly without any prior deformation of the specimen. Since in civil engineering a more gradual failure is more favourable, the TL1 laminates are preferred above the other two TL's. 


\section{Discussion}

Numerical simulations (Figure 8) confirm the decrease in global stiffness in relation with an increasing stacking angle. Further these models also approve the increased local stiffness at the location of an overlap. However, the models assume a gradual decrease in local stiffness between two overlaps, which can be refuted by the experimental results shown in Figure 4. Here the local stiffness between two overlaps is roughly consistent such as for a PP laminate. Finally, the failure mechanism observed in the tests for the TL's does not correspond to the failure mechanism presented in

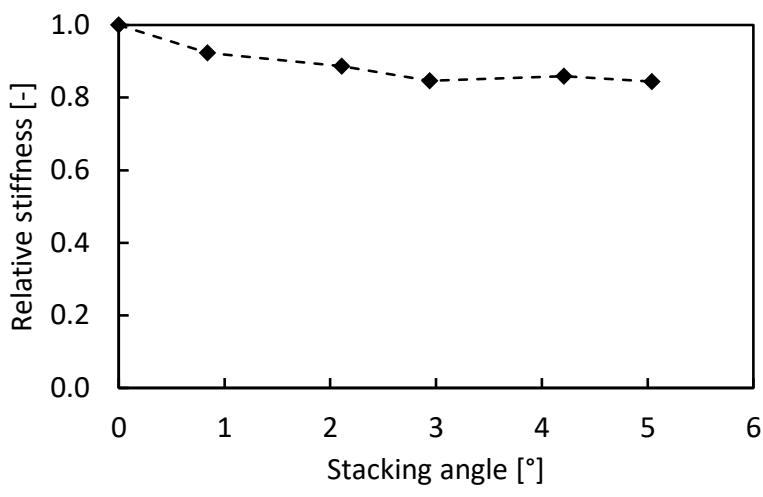

Figure 8: Numerical results of relative stiffness in function of the stacking angle literature, seen in Figure 1. Failure occurs by shear between two different stacks instead of ply failure perpendicular to the loading direction. This observation can be confirmed by numerical simulations of an uniaxial tensile test of the TL's.

In Figure 4 (d) and (f) a local stiffness decrease is present at the places of the overlap, because there is a small misalignment between the stacks. This flaw is specific to the VARTM production process of the laminates, since the layup of the different layers and stacks occurs by hand. The same can be said about the excessive peak stiffness at about $35 \mathrm{~mm}$ in Figure 4 (d), originating from an accumulation of stacks.

Through these experimental findings it is possible to evaluate, adjust and validate the numerical simulations and establish adequately validated models on laminate level. These laminate models can be adopted as input for numerical models of a unit cell of the tiled sandwich panel. These models can then be further expanded to a whole tiled sandwich panel. The results will make it able to formulate a better theoretical and experimental basis for applications of TC's, prove the performance claimed for FRP infrastructures and to better formulate new product and process developments. Using this knowledge, applications in other sectors, such as aerospace technology, offshore and shipbuilding, defence, energy industry and even in the far future at molecular levels (Z- and U-folded graphene micro-structures) are opened up.

\section{Conclusion}

In this study the difference between the global and local stiffness of tiled laminates is demonstrated and analysed in comparison with a plane-parallel reference sample, for different stacking angle using the full strain field obtained from DIC measurements. It was found that the global stiffness only slightly decreases while the total failure strength reduces significantly in relation with an increasing stacking angle. Although this reduction is significant at approximately $50 \%$ in these tests, the authors are convinced that in a primarily stiffness, robustness [5] and ease of construction based design, this reduction is still acceptable.

\section{References}

[1] M. Veltkamp and J. Peeters, Hybrid Bridge Structure Composed of Fibre Reinforced Polymers and Steel, Structural Engineering International, 24(3), 2014, pp. 425-427.

[2] Fibercore Europe (2010). Report FCE-1001: Material properties used in InfraCore Structures. FCE.

[3] American Society for Testing and Materials (2002). Standard Test Method for Tensile Properties of Polymer Matrix Composite Materials.

[4] McCormick N., Lord J. (2010). Digital Image Correlation. Materials Today, Volume 13, Issue 12, Pages 52-54. 
[5] European Committee for Standardization, EN 1990: 2002 - Eurocode - Basis of structural design, consolidated version, including A1:2005 and AC:2010, CEN, Brussels, 2015. 